\title{
Rosiglitazone attenuates myocardial remodeling in spontaneously hypertensive rats
}

\author{
Yun $\mathrm{Ti}^{1,2,3}$, Ming-Xiu Hao ${ }^{1,2,3}$, Chuan-Bao $\mathrm{Li}^{1}$, Zhi-Hao Wang ${ }^{1,2}$, Xiao-Yang Hou ${ }^{1,2}$, Xue-Qiang Zhao ${ }^{1,2}$, \\ Jun-Ni Liu ${ }^{1,2}$, Wei Zhang ${ }^{1,2}$, Yun Zhang ${ }^{1,2}$ and Pei-Li Bu ${ }^{1,2}$ \\ Rosiglitazone, an important peroxisome proliferator-activated receptor- $\gamma$ (PPAR- $\gamma$ ) agonist, improves left ventricular (LV) \\ hypertrophy in diet-induced hypercholesterolemic rats. However, the effects and underlying mechanisms of rosiglitazone on \\ myocardial remodeling in spontaneous hypertension rats (SHRs) are unclear. Twenty male 8-week-old SHRs were randomly \\ divided into two groups: one treated with oral saline $(n=10)$ and the other treated with rosiglitazone $\left(5 \mathrm{mg} \mathrm{kg}^{-1}\right.$ day $\left.^{-1}, n=10\right)$. \\ Ten age-matched Wistar-Kyoto rats were selected as a normal control group. Echocardiography, immunohistochemistry, real-time \\ reverse transcriptase-PCR and western blot analysis were performed to assess the effects of rosiglitazone. After 16 weeks \\ of treatment, LV hypertrophy was significantly attenuated by rosiglitazone (LV weight/body weight, $2.35 \pm 0.11 \mathrm{vs}$. \\ $2.56 \pm 0.14 \mathrm{mg} \mathrm{g}^{-1}$ ). According to the echocardiography results, thickening of the LV wall was reduced, and mid-wall fractional \\ shortening was improved by rosiglitazone. Similarly, the excessive collagen deposition and upregulation of collagen I and \\ collagen III seen in SHRs receiving saline were significantly attenuated in SHRs receiving rosiglitazone. In addition, rosiglitazone \\ treatment increased the activity of matrix metalloproteinase-9 (MMP-9) and normalized the MMP-9/tissue inhibitor of \\ metalloproteinase-1 ratio. Furthermore, activator protein-1 (AP-1) activation and nuclear factor-kappa B (NF-kB) expression \\ were suppressed in the rosiglitazone-treated group. These results demonstrate that the PPAR- $\gamma$ agonist rosiglitazone had \\ beneficial effects on myocardial remodeling in SHRs by way of decreasing AP-1 activation and NF-KB expression, which \\ may help in further inhibiting transcription of the downstream genes involved in the pathogenesis of myocardial remodeling \\ induced by hypertension.
}

Hypertension Research (2011) 34, 354-360; doi:10.1038/hr.2010.242; published online 20 January 2011

Keywords: myocardial remodeling; peroxisome proliferator-activated receptor; rosiglitazone

\section{INTRODUCTION}

Hypertension is frequently associated with cardiac fibrosis and left ventricular (LV) hypertrophy. Cardiac remodeling is an important adaptive physiological response to hemodynamic overload to the heart that results in the excessive accumulation of collagen fibers. Simultaneously, myocardial remodeling is accompanied by an imbalance between matrix metalloproteinases (MMPs) and tissue inhibitors of metalloproteinases (TIMPs). The breakdown of collagen is affected by MMPs, which in turn are influenced by TIMPs. The interaction between MMPs and TIMPs may be modulated by many points, each of which may affect the final outcome of the fibrous process in the heart. Previous studies ${ }^{1}$ have also suggested that MMP-9 and TIMP-1 have an important role during cardiac remodeling. The promoter regions of MMP-9 and TIMP-1 genes contain several functional regulatory motifs that can bind with several well-characterized transcription factors, including nuclear factor-kappa B (NF- $\kappa \mathrm{B})$ and activator protein-1 (AP-1). AP-1 complexes most often consist of c-Fos and c-Jun, which can form functional AP-1. Heterodimerization of c-Fos with c-Jun results in a more stable AP-1 complex that increases the capacity of c-Jun to transactivate target genes. ${ }^{2}$ $\mathrm{Li}$ et $a .^{3}{ }^{3}$ demonstrated that stimulation of the AP-1 and NF- $\mathrm{BB}$ pathways mediates hepatic fibrogenesis induced by intrahepatic RAAS. Thus, the AP-1 (c-Fos/c-Jun heterodimers) and NF- $\kappa \mathrm{B}$ pathways may participate in the process of fibrosis.

Peroxisome proliferator-activated receptor- $\gamma$ (PPAR- $\gamma$ ), one of the three members of a superfamily of ligand-activated transcription factors, has a crucial role in the regulation of insulin sensitivity and diabetic complications. A PPAR- $\gamma$ agonist inhibits cardiac hypertrophy in cardiac myocytes. ${ }^{4}$ Rosiglitazone, a ligand of PPAR- $\gamma$, represses AP- 1 and NF- $\kappa \mathrm{B}$ transcriptional activity and improves LV hypertrophy in diet-induced hypercholesterolemic rats. ${ }^{5}$ In the present study, we used spontaneous hypertension rats (SHRs) to test the hypothesis that

${ }^{1}$ Key Laboratory of Cardiovascular Remodeling and Function Research, Chinese Ministry of Education and Chinese Ministry of Health, Shandong University Qilu Hospital, Shandong, China and ${ }^{2}$ Department of Cardiology, Shandong University Qilu Hospital, Shandong, China

3These authors contributed equally to this work.

Correspondence: Dr P-L Bu, Key Laboratory of Cardiovascular Remodeling and Function Research, Chinese Ministry of Education and Chinese Ministry of Health, Shandong University Qilu Hospital, No. 107, Wen Hua Xi Road, Jinan, Shandong 250012, China.

E-mail: peilibu@hotmail.com

Received 17 June 2010; revised 17 September 2010; accepted 20 September 2010; published online 20 January 2011 
rosiglitazone may affect cardiac remodeling by modulating AP-1 and NF- $\kappa \mathrm{B}$ and, furthermore, by regulating MMP-9 and TIMP-1 expression, which affects collagen accumulation.

\section{METHODS}

\section{Animals and study design}

This study was conducted in accordance with the recommendations of the Institutional Animal Care and Use Committee of Shandong University. Twenty 8-week-old male SHRs and ten 8-week-old male Wistar-Kyoto (WKY) rats, purchased from Wei Tong Li Hua (Beijing, China), were used in this study. Animals were housed in a temperature-controlled $\left(22 \pm 0.5^{\circ} \mathrm{C}\right)$ room and were fed standard rodent chow and water ad libitum. A 12-h/12-h light/dark cycle was maintained. SHRs were randomly divided into two groups: one group was treated with oral saline $(n=10)$ and the other group was treated with oral rosiglitazone (4 mg per tablet, GlaxoSmithKline, Middlesex, UK) dissolved in $0.9 \%$ saline at a dose of $5 \mathrm{mg} \mathrm{kg}^{-1} \mathrm{day}^{-1}(n=10)$. The rosiglitazone dose was based on previous studies and our preliminary experiments. Age-matched WKY rats $(n=10)$ were treated with oral saline as a control group. Rosiglitazone or saline was delivered by gavage at the same time once a day for 16 weeks. Echocardiography was performed at the beginning and at the end of the experiment. After 16 weeks of treatment, heart rate, systolic blood pressure and diastolic blood pressure were measured with a noninvasive tail-cuff system (Softron BP-98A, Tokyo, Japan) as previously described. ${ }^{6}$ After transthoracic echocardiography was performed, animals were anesthetized with pentobarbital, and body weight (BW) and LV weight (LVW) were determined. The heart was either (1) fixed in $10 \%$ formaldehyde and then paraffin-embedded for histopathological analysis and immunohistochemical staining or (2) snap frozen in liquid nitrogen and stored at $-80{ }^{\circ} \mathrm{C}$ for real-time PCR and western blot analysis.

\section{Echocardiography}

The rats were anesthetized with pentobarbital (2.5\%), and the anterior chest was shaved. Echocardiography was performed with a Philips 7500 Ultrasound System (Philips Medical Systems, Andover, MA, USA), using a $12.0-\mathrm{MHz}$ transducer. Images were obtained from M-mode, two-dimensional (2-D), pulse wave (PW) Doppler and acoustic density. To optimize the image, a transmission gel was used between the transducer and the animal's chest. All measurements nuclear magnetic resonance were performed by the same observer based on the average of six consecutive cardiac cycles. Wall thickness and LV dimensions were obtained from a long-axis view at the level of chordae tendineae. Specific dimensions included LV end-diastolic diameter (LVIDd) and end-diastolic posterior and interseptum wall thickness (LVPWd and IVSd, respectively). The LV ejection fraction was calculated according to Teichholz's formula [(LVEDV-LVESV)/LVEDV $\times 100 \%$ ], where LVEDV and LVESV are the end-diastolic and end-systolic volumes, respectively. Mid-wall fractional shortening was calculated as [(LVIDd+PWd/2+IVSd/2) $-($ LVIDs+PWs/2+IVSs/2)]/ (LVIDd+PWd/2+IVSd/2), where LVIDs is the LV end-systolic diameter and LVPWs and IVSs the end-systolic posterior wall and interseptum thickness, respectively. The mitral valve pulsed Doppler recordings were obtained from the apical four-chamber view. After pulsed Doppler, transmitral flow velocity parameters were evaluated, including peak $E$, peak $A$ and the $E / A$ ratio.

Acoustic densitometry was performed at the interventricular septum and posterior wall of the LV using an ultrasonic imaging system (Philips 7500, Philips Technologies), operating in the $\mathrm{AD}$ acquisition mode, which permits the acquisition, display and analysis of real-time integrated backscatter (IBS) images on the basis of a previously described technique. ${ }^{7}$ The following parameters were measured: time-averaged IBS, cyclic variation of IBS and standardized IBS (IBS\%).

\section{Tissue preparation and morphometric analysis}

Hearts were fixed in $4 \%$ paraformaldehyde, embedded in paraffin, and sectioned at $5-\mu \mathrm{m}$ intervals. Hematoxylin and eosin staining and Masson's trichrome staining were performed using standard procedures. The collagen volume fraction and perivascular collagen area/luminal area were determined by quantitative morphometry with an automated image analysis (Image-Pro Plus, Version 5.0, Media Cybernetics, Silver Spring, MD, USA). The cardiac collagen volume fraction was calculated as the ratio of the sum of total interstitial collagen area to the sum of total connective tissue and muscle area in the entire visual field of the section, as reported previously. ${ }^{8}$ Perivascular collagen was excluded from the measurement of collagen volume fraction. To normalize the area of perivascular collagen around vessels with different sizes, the perivascular collagen content was represented as the perivascular collagen area-to-luminal area ratio. Measurements of all parameters from the three heart sections per rat were averaged.

\section{TEM}

LV myocardial tissues were fixed with $2 \%$ glutaraldehyde overnight and washed three times with $0.2 \mathrm{moll}^{-1}$ phosphate buffer, re-fixed with $1 \%$ osmium tetraoxide, washed with $0.2 \mathrm{moll}^{-1}$ phosphate buffer again and dehydrated through an ethanol series. The samples were immersed in Epon812 resin/ acetone (1:1) for $30 \mathrm{~min}$, immersed in fresh Epon 812 resin for $30 \mathrm{~min}$ and then embedded for convergence overnight at $70^{\circ} \mathrm{C}$. Ultrathin sectioning was performed using a microtome (LKB 8800, LKB Produkter AB, Bromma, Sweden). Embedded sections were stained with lead citrate and observed by a transmission electron microscope (TEM, H-7000FA, Hitachi, Tokyo, Japan).

\section{Immunohistochemical staining}

Immunohistochemistry was carried out with primary antibodies (c-Fos, c-Jun and NF-кB P65 immunoglobulin G; Abcam, Cambridge, UK). Sections were deparaffinized, washed with phosphate-buffered saline and incubated with $3 \%$ $\mathrm{H}_{2} \mathrm{O}_{2}$. After antigen microwave restoration, non-specific binding was blocked with $5 \%$ normal goat serum. Sections were incubated overnight with anti-c-Jun (diluted 1:200), anti-c-Fos (diluted 1:100) and anti-NF- $\mathrm{KB}$ P65 immunoglobulin G (diluted 1:100) antibodies in a humidified chamber at $4{ }^{\circ} \mathrm{C}$. After the sections were incubated with a secondary-conjugated immunoglobulin $\mathrm{G}$ antibody for $1 \mathrm{~h}$ at $37^{\circ} \mathrm{C}$ and two additional washing steps were performed with phosphate-buffered saline, slides were developed with diaminobenzidine (Dako, Glostrup, Denmark) for $5 \mathrm{~min}$ in the dark and counterstained with hematoxylin. All sections were examined using a light microscope (Olympus, Tokyo, Japan) and assessed using a JD-801 computer-aided image analyzer.

\section{Real-time quantitative PCR}

Total RNA was isolated from frozen myocardial tissues using TRIzol reagent (Invitrogen, Carlsbad, CA, USA). The quality of RNA was determined using spectrophotometry (DU 800, Beckman, Palo Alto, CA, USA). Real-time reverse transcriptase-PCR was then performed with the SYBR Green PCR kit (Perfect Real Time; TaKaRa, Kyoto, Japan) according to the manufacturer's instructions. The PCR conditions were as follows: $95^{\circ} \mathrm{C}$ for $10 \mathrm{~s}, 60^{\circ} \mathrm{C}$ for $5 \mathrm{~s}$ and $72{ }^{\circ} \mathrm{C}$ for $10 \mathrm{~s}$ for MMP-9; $95^{\circ} \mathrm{C}$ for $10 \mathrm{~s}, 57^{\circ} \mathrm{C}$ for $5 \mathrm{~s}$ and $72^{\circ} \mathrm{C}$ for $5 \mathrm{~s}$ for collagen I; $95^{\circ} \mathrm{C}$ for $10 \mathrm{~s}, 55^{\circ} \mathrm{C}$ for $5 \mathrm{~s}$ and $72^{\circ} \mathrm{C}$ for $15 \mathrm{~s}$ for collagen III; $95^{\circ} \mathrm{C}$ for $10 \mathrm{~s}, 62^{\circ} \mathrm{C}$ for $5 \mathrm{~s}$ and $72^{\circ} \mathrm{C}$ for $10 \mathrm{~s}$ for c-Jun and TIMP- $1 ; 95^{\circ} \mathrm{C}$ for $10 \mathrm{~s}, 54^{\circ} \mathrm{C}$ for $5 \mathrm{~s}$ and $72^{\circ} \mathrm{C}$ for $10 \mathrm{~s}$ for c-Fos; and $95^{\circ} \mathrm{C}$ for $10 \mathrm{~s}, 58^{\circ} \mathrm{C}$ for $5 \mathrm{~s}$ and $72^{\circ} \mathrm{C}$ for $10 \mathrm{~s}$ for PPAR- $\gamma$ and NF-KB. Melting curve analysis is usually performed immediately after realtime PCR to identify nonspecific products. The PCR efficiency was assessed with serial dilutions of a sample of cDNA from the normal control group. All reactions were performed in duplicate, and the data were analyzed using LightCycler software 4.0 (Roche Diagnostic Systems, Inc., Branchburg, NJ, USA).

\section{Gelatinase zymography}

To determine the activity of MMP-9, zymographic analysis was performed as previously described. The samples were subjected to electrophoresis in polyacrylamide gels containing $1 \mathrm{mg} \mathrm{ml}^{-1}$ gelatin in the presence of sodium dodecyl sulfate, washed twice in $2.5 \%$ Triton X-100 for $40 \mathrm{~min}$, rinsed briefly, and incubated at $37^{\circ} \mathrm{C}$ for $42 \mathrm{~h}$ in $100 \mathrm{mmoll}^{-1}$ Tris- $\mathrm{HCl}\left(\mathrm{pH}\right.$ 7.4) and $10 \mathrm{mmoll}^{-1}$ $\mathrm{CaCl}_{2}$. Then the samples were stained with Coomassie Brilliant Blue R-250 (Amresco Inc, Solon, OH, USA) and destained in a solution of $7.5 \%$ acetic acid and 5\% methanol. Zones of enzymatic activity appeared as clear bands against a black background; they were measured using a computer image analysis system.

\section{Co-immunoprecipitation and western blot analysis}

Western blot analysis was carried out with primary antibodies (TIMP-1, c-Fos, c-Jun and NF-кB P65 immunoglobulin G; Abcam). Co-immunopreci- 
pitation assays were used to evaluate the interaction of c-Fos with c-Jun. Co-immunoprecipitation was carried out with anti-c-Fos, and the proteins were analyzed using western blot with an anti-c-Jun antibody. Samples containing equal amounts of proteins were separated on polyacrylamide gels with a minivertical electrophoresis system using a standard method. After electrophoresis, proteins were transferred onto polyvinyldifluoridene membranes. The membranes were then incubated with primary antibodies overnight at $4{ }^{\circ} \mathrm{C}$. Then, horseradish peroxidase-conjugated second antibodies were applied for $2 \mathrm{~h}$ at room temperature. The proteins were visualized using the Chemiluminescent Substrate Kit (Solarbio, Beijing, China), and quantified using the UN-SCAN-IT gel Automated Digitizing System (UN-SCAN-IT gel software, Silk Scientific, Inc., Orem, UT, USA). Measurements were expressed as arbitrary units. The results were normalized against $\beta$-actin.

\section{Statistical analysis}

Data are presented as means \pm s.e.m. We used SPSS 16.0 for statistical analyses. The differences between all measured values were assessed by one-way analysis of variance followed by post-hoc analysis with Tukey's test. $P<0.05$ was considered statistically significant.

\section{RESULTS}

\section{BP and cardiac morphology}

The characteristics of the three groups at week 1 and week 16 are given in Table 1. After treatment with rosiglitazone, an increase in BW was observed in the rosiglitazone-treated group $(P<0.05 v s$. SHRs). In contrast, systolic blood pressure was significantly decreased com- pared with SHRs $(P<0.01$ vs. SHRs). Concomitant with the blood pressure decrease, both the LVW and the LVW/BW ratio in the rosiglitazone-treated group became significantly lower than those of the age-matched SHRs $(P<0.05$ vs. SHRs $)$. However, diastolic blood pressure and heart rate were not affected by rosiglitazone.

\section{Effects of rosiglitazone on LV structure and function}

To investigate whether rosiglitazone administration would affect in vivo cardiac function, cardiac performance was analyzed by echocardiography (Table 2, Figure 1). Echocardiographic examination of the LV confirmed our previous findings. After 1 week of the experiment, LVPWd and IVSd were significantly higher in both SHR groups than in the WKY group (Table 2). LVPWd and IVSd significantly increased, accompanied by an elevated LVW/BW ratio, in SHRs compared with WKY at week $16(P<0.05$ vs. WKY) (Figure 1a). However, LVIDd decreased in SHRs compared with WKY $(P<0.05$ vs. WKY) (Figures la and d). After treatment with rosiglitazone, IVSd and LVPWd markedly decreased; in contrast, LVIDd increased compared with SHRs $(P<0.01 v s$. SHR) (Figures 1a and d). Mid-wall fractional shortening and $E / A$ were used to investigate the temporal changes of systolic and diastolic function, respectively. Mid-wall fractional shortening and $E / A$ decreased significantly in SHRs; these parameters were significantly improved at week 16 in SHRs treated with rosiglitazone (Figures 1b, e and f). Additionally, SHRs receiving saline at week 16 showed significantly increased standardized IBS and decreased cyclic

Table 1 Animal characteristics at week 1 and week 16 after Ros treatment

\begin{tabular}{|c|c|c|c|c|c|c|}
\hline & \multicolumn{3}{|c|}{ Week 1} & \multicolumn{3}{|c|}{ Week 16} \\
\hline & $W K Y$ & SHR & Ros & $W K Y$ & SHR & Ros \\
\hline HR (b.p.m.) & $439 \pm 28$ & $438 \pm 29$ & $432 \pm 46$ & $395 \pm 24$ & $392 \pm 18$ & $389 \pm 20$ \\
\hline $\mathrm{SBP}(\mathrm{mm} \mathrm{Hg})$ & $119 \pm 7$ & $165 \pm 5^{\star *}$ & $164 \pm 4^{* *}$ & $117 \pm 7$ & $196 \pm 3^{* * *}$ & $182 \pm 4^{* \star * \ddagger}$ \\
\hline $\mathrm{DBP}(\mathrm{mm} \mathrm{Hg})$ & $96 \pm 15$ & $146 \pm 9^{* * *}$ & $148 \pm 6^{* * *}$ & $97 \pm 14$ & $367 \pm 7^{*}$ & $158 \pm 9^{* * *}$ \\
\hline BW (g) & $241 \pm 13$ & $198 \pm 12^{*}$ & $204 \pm 9^{* *}$ & $552 \pm 11$ & $162 \pm 8^{* * *}$ & $379 \pm 10^{*} \dagger$ \\
\hline LVW (mg) & & & & $788 \pm 53$ & $940 \pm 36^{*}$ & $893 \pm 36^{*} \dagger$ \\
\hline LVW/BW (mg g $\left.{ }^{-1}\right)$ & & & & $1.43 \pm 0.08$ & $2.56 \pm 0.14^{*}$ & $2.35 \pm 0.11^{* \dagger}$ \\
\hline
\end{tabular}

Abbreviations: DBP, diastolic blood pressure; HR, heart rate; LVW, left ventricular weight; LVW/BW, left ventricular weight/body weight ratio; Ros, rosiglitazone-treated group; SBP, systolic blood pressure; SHR, spontaneous hypertension rat; WKY, Wistar-Kyoto.

Values are mean \pm s.e.m.

${ }^{*} P<0.01,{ }^{* *} P<0.05,{ }^{* * *} P<0.001$ vs. WKY; ${ }^{\dagger} P<0.05, \stackrel{\ddagger}{\ddagger}<0.01$ vs. SHR.

Table 2 Echocardiographic data in three groups of rats at week 1 and week 16

\begin{tabular}{|c|c|c|c|c|c|c|}
\hline & \multicolumn{3}{|c|}{ Week 1} & \multicolumn{3}{|c|}{ Week 16} \\
\hline & $W K Y$ & SHR & Ros & $W K Y$ & SHR & Ros \\
\hline LVIDd (mm) & $5.28 \pm 0.07$ & $5.26 \pm 0.03$ & $5.25 \pm 0.04$ & $6.12 \pm 0.07$ & $5.84 \pm 0.03 *$ & $5.95 \pm 0.05^{\star \ddagger}$ \\
\hline IVSd (mm) & $1.34 \pm 0.02$ & $1.69 \pm 0.04^{* *}$ & $1.69 \pm 0.03^{* *}$ & $1.75 \pm 0.03$ & $2.15 \pm 0.04^{*}$ & $2.07 \pm 0.03 * \neq$ \\
\hline LVPWd (mm) & $1.33 \pm 0.04$ & $1.69 \pm 0.05^{* *}$ & $1.70 \pm 0.03^{* *}$ & $1.73 \pm 0.03$ & $2.15 \pm 0.05^{*}$ & $2.08 \pm 0.05^{* \ddagger}$ \\
\hline LVEF (\%) & $79.75 \pm 0.23$ & $79.85 \pm 0.14$ & $79.77 \pm 0.09$ & $79.75 \pm 0.11$ & $79.68 \pm 0.23$ & $79 . .81 \pm 0.15$ \\
\hline MFS (\%) & $24.75 \pm 0.33$ & $24.73 \pm 0.24$ & $24.77 \pm 0.23$ & $24.69 \pm 0.31$ & $23.33 \pm 0.44^{*}$ & $23.82 \pm 0.23^{* \ddagger}$ \\
\hline$E / A$ & $2.01 \pm 0.03$ & $2.00 \pm 0.04$ & $2.01 \pm 0.03$ & $2.01 \pm 0.03$ & $1.95 \pm 0.02 *$ & $1.97 \pm 0.02^{* \dagger}$ \\
\hline IBS\% \% & $1.82 \pm 0.03$ & $1.81 \pm 0.06$ & $1.81 \pm 0.04$ & $1.83 \pm 0.08$ & $2.42 \pm 0.19$ * & $2.27 \pm 0.12^{* \dagger}$ \\
\hline $\mathrm{CVIB}_{\text {IVS }}(\mathrm{dB})$ & $10.49 \pm 0.15$ & $10.46 \pm 0.34$ & $10.48 \pm 0.28$ & $10.78 \pm 0.48$ & $9.21 \pm 0.28^{*}$ & $9.61 \pm 0.34^{* \dagger}$ \\
\hline IBS\%LVPW & $1.81 \pm 0.06$ & $1.81 \pm 0.03$ & $1.80 \pm 0.10$ & $1.80 \pm 0.10$ & $2.30 \pm 0.14^{*}$ & $2.13 \pm 0.12^{* \dagger}$ \\
\hline CVIB & $10.66 \pm 0.23$ & $10.65 \pm 0.37$ & $10.63 \pm 0.05$ & $10.68 \pm 0.60$ & $9.31 \pm 0.35^{*}$ & $9.77 \pm 0.25^{* \dagger}$ \\
\hline
\end{tabular}

Abbreviations: CVIB, cyclic variation of IBS; EIA, ratio of the early to the late peak diastolic transmitral flow velocity; IBS, integrated backscatter; IVSd, end-diastolic septal thickness; LVEF, left ventricular ejection fraction; LVIDd, left ventricular end-diastolic diameter; LVPWd, end-diastolic posterior wall thickness; MFS, midwall fractional shortening; Ros, rosiglitazone-treated group; SHR, spontaneous hypertension rat; WKY, Wistar-Kyoto.

SHR, spontaneous hyperte
Values are mean \pm s.e.m.

${ }^{*} P<0.05,{ }^{* *} P<0.01$ vs. WKY; ${ }^{\dagger} P<0.05,{ }^{\ddagger} P<0.01$ vs. SHR. 

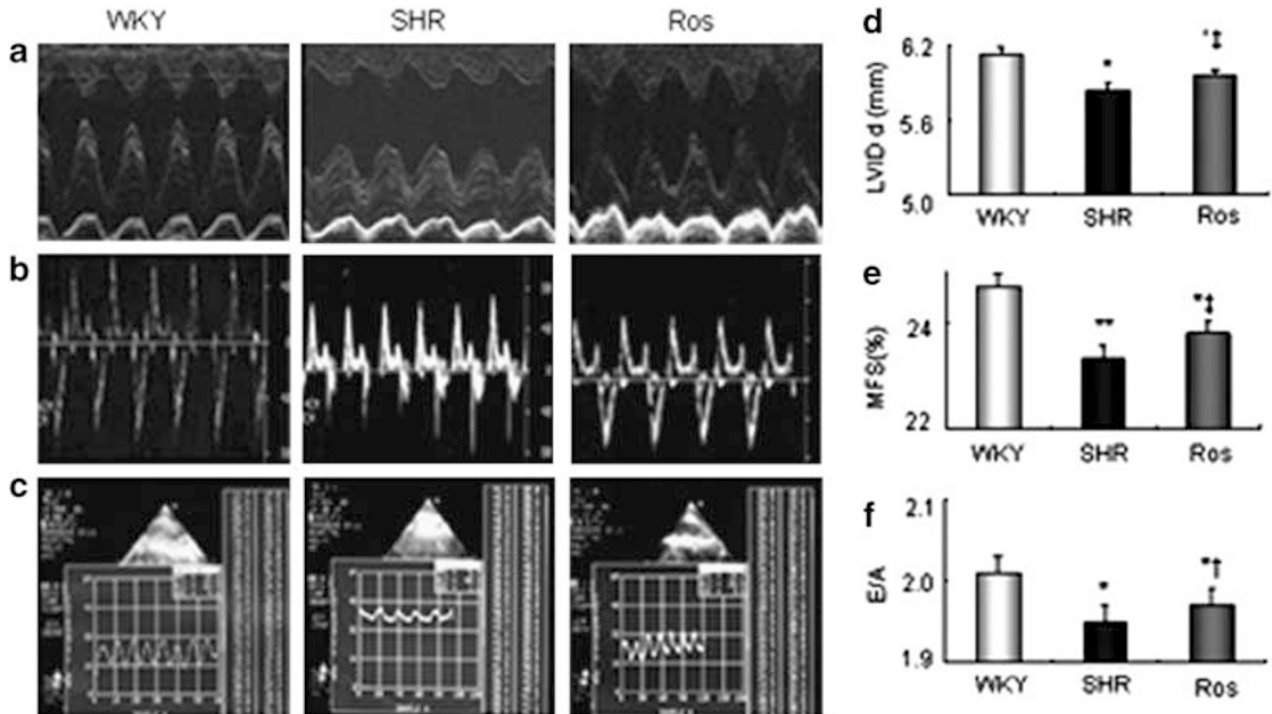

Figure 1 Rosiglitazone administration improves cardiac function in spontaneous hypertension rats (SHRs). (a) Representative M-mode echocardiogram showing improved wall motion after treatment. (b) Representative pulsed-wave Doppler echocardiogram of mitral inflow showing improved E/A. (c) Representative AD image showing normalized standardized IBS and cyclic variation of IBS. (d-f) After 16 weeks of treatment, the left ventricular end-diastolic diameter (LVIDd), mid-wall fractional shortening (MFS) and $E / A$ were improved in the rosiglitazone-treated group (Ros). ${ }^{*}<0.05$, ${ }^{*} P<0.01$ vs Wistar-Kyoto (WKY) rats; ${ }^{\dagger} P<0.05, \ddagger P<0.01$ vs. SHRs.
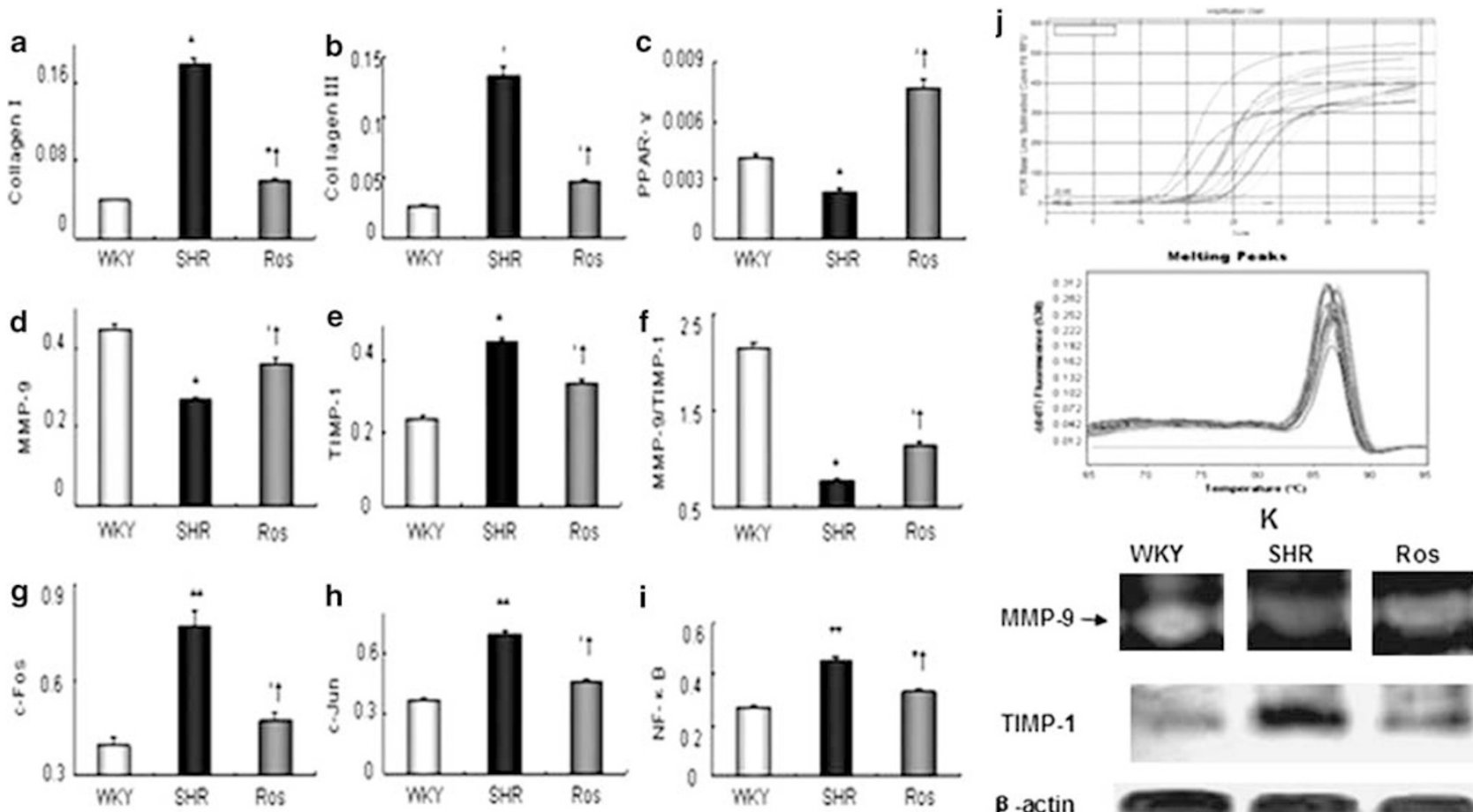

TIMP.1

及 -actin

Figure 2 (a-i) Expression of cardiac fibrotic markers, peroxisome proliferator-activated receptor- $\gamma$ (PPAR- $\gamma$ ), c-Fos, c-Jun and nuclear factor-kappa B (NF- $\mathrm{B}$ ), detected by real-time reverse transcriptase (RT)-PCR 16 weeks after rosiglitazone administration ( $n=5$ per group). (j) Amplification chart and the melting peaks of the RT-PCR results. (k) Cardiac matrix metalloproteinase-9 (MMP-9) and tissue inhibitor of metalloproteinase-1 (TIMP-1) levels detected by gelatinase zymography and western blot analysis ( $n=5$ per group). Values indicate the expression level relative to the Wistar-Kyoto (WKY) group ( \pm s.e.m.). ${ }^{*} P<0.05,{ }^{*} P<0.01$ vs. WKY; ${ }^{\dagger} P<0.05$ vs. spontaneous hypertension rats (SHRs).

variation of IBS in the LV posterior walls and interventricular septum in comparison with their week 1 levels and the WKY group, whereas rosiglitazone treatment substantially improved these parameters in SHRs measured at week 16 (Table 2, Figure 1c).

\section{Myocardial PPAR- $\gamma$ expression}

Rosiglitazone-treated hearts exhibited a $>3$-fold increase in PPAR- $\gamma$ expression compared with untreated SHR hearts $(0.0077 \pm 0.0013$ vs. $0.0024 \pm 0.0009$, respectively, $P<0.05$ ) (Figure $2 \mathrm{c}$ ). 
Effect of rosiglitazone on pathological characteristics, collagen deposition, and collagen I and III mRNA expression

Histological examination of cardiac tissues demonstrated severe myocyte hypertrophy, disarray and extensive interstitial fibrosis in SHRs compared with WKY (Figures 3a1-a3, b1-b3). TEM analysis showed extensive muscle fiber loss and vacuolar change within individual fibers in hearts from SHRs (Figures 3d1-d3). The pathological changes were significantly improved by rosiglitazone administration. The effect of rosiglitazone on cardiac fibrosis was identified by Masson's trichrome staining (Figures 3b1-b3, c1-c3). Treatment with rosiglitazone markedly attenuated the increase in collagen volume fraction and perivascular collagen area-to-luminal area ratio compared with SHRs $(P<0.05$ vs. SHR) (Figures 3b1-b3, c1-c3). These observations were confirmed by quantitative analysis (Figures $3 \mathrm{e}$ and $\mathrm{f}$ ). Rosiglitazone ameliorated the increased collagen I and III mRNA expression, which was paralleled by histological findings of decreased interstitial and perivascular fibrosis compared with untreated SHRs (Figures $2 \mathrm{a}$ and $\mathrm{b}$ ).

\section{Myocardial levels of MMP-9 and TIMP-1}

To examine the potential mechanisms through which rosiglitazone may exert beneficial effects on matrix turnover and fibrosis, we examined myocardial MMP-9 and TIMP-1 expression. The MMP-9 mRNA level was elevated by treatment with rosiglitazone $(P<0.05$ vs. SHRs). In contrast, rosiglitazone remarkably reduced the TIMP-1
mRNA level $(P<0.05$ vs. SHRs). Thus, in the treated group, rosiglitazone significantly elevated the MMP-9/TIMP-1 ratio $(P<0.05$ vs. SHRs) (Figures 2d-f). Additionally, the activity of MMP-9, as assessed by gelatinase zymography, was elevated, and the protein expression level of TIMP-1 was inhibited by treatment with rosiglitazone (Figure 2k).

\section{Rosiglitazone decreased c-Fos, c-Jun, c-Fos/c-Jun heterodimers (AP-1) and NF-кB expression}

The promoters of MMP-9 and TIMP-1 contain two important transcriptional elements, which are the binding sites of AP-1 and NF- $\kappa \mathrm{B}$. To further determine the involvement of the activation of AP- 1 or NF- $\kappa B$ in stimulating MMP-9 expression induced by rosiglitazone, the mRNA levels of c-Fos, $\mathrm{c}-J$ un and NF- $\kappa \mathrm{B}$ were detected. Rosiglitazone remarkably ameliorated the increased mRNA expression of c-Fos, c-Jun and NF- $\mathrm{KB}(P<0.05 v s$. SHRs) (Figure $2 \mathrm{~g}-\mathrm{i})$. Furthermore, rosiglitazone reduced the protein level of c-Jun, AP-1 (c-Jun/ c-Fos heterodimers) and NF- $\kappa \mathrm{B}$ according to immunohistochemical staining and western blot analysis $(P<0.05 v s$. SHRs) (Figure 4$)$.

\section{DISCUSSION}

In the present study, we demonstrated that treating SHRs with the PPAR- $\gamma$ agonist rosiglitazone improved LV hypertrophy. Moreover, rosiglitazone remarkably decreased collagen I and III expression. Reduced fibrosis was associated with rosiglitazone-mediated changes
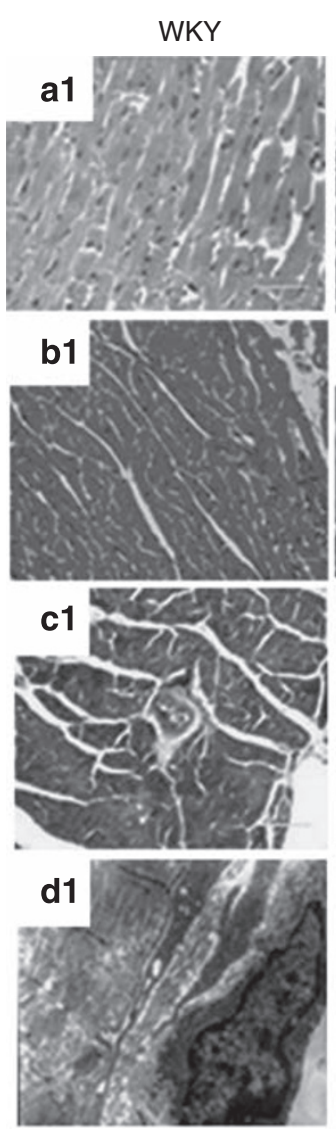

SHR
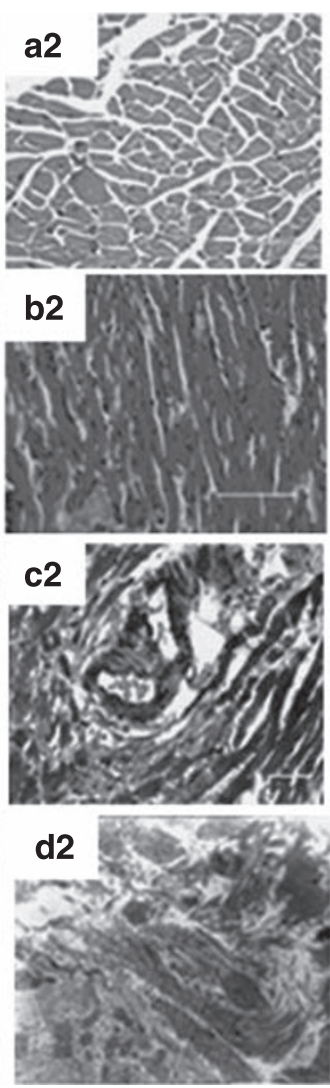
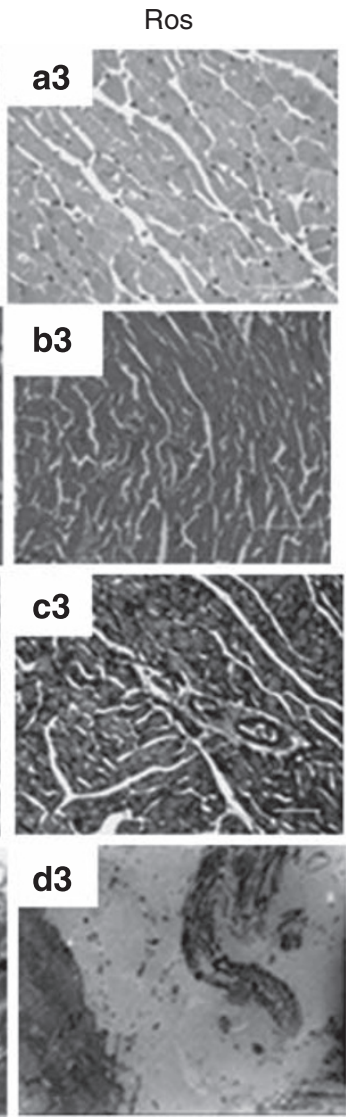
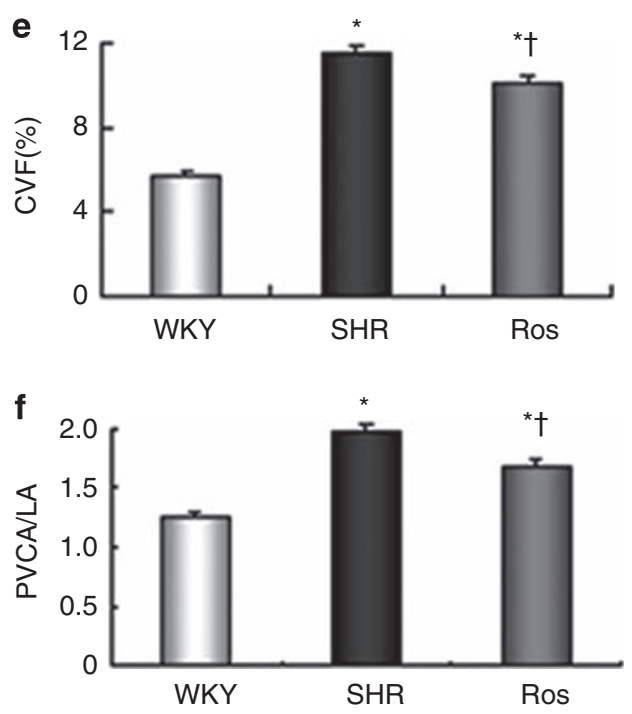

Figure 3 Rosiglitazone administration suppressed myocardial remodeling in spontaneous hypertension rats (SHRs). Tissue sections were stained with hematoxylin and eosin $(H \& E)(\mathbf{a} 1-\mathbf{a} 3 ; \times 400)$ and Masson's trichrome staining $(\mathbf{b} 1-\mathbf{b} 3, \mathbf{c} 1-\mathbf{c} 3 ; \times 200)$. The scale bar represents $100 \mu \mathrm{m}$. Ultrastructure of cardiac sections $(\mathbf{d} 1-\mathbf{d} 3 ; \times 8000)$. (e) Quantitative analysis of the ratio of myocardial fibrosis area to total myocardial area (percentage). (f) Quantitative analysis of the ratio of perivascular fibrosis area to luminal area. Fibrosis areas were quantified by image analysis using Masson's trichrome staining. At least six areas were evaluated in each section, and each value represents the mean \pm s.e.m. ( $n=5$ in each group). ${ }^{*} P<0.05$ vs. Wistar-Kyoto (WKY) rats; ${ }^{\dagger} P<0.05$ vs. SHRs. CVF, collagen volume fraction; PVCA/LA, perivascular collagen area/luminal area. 


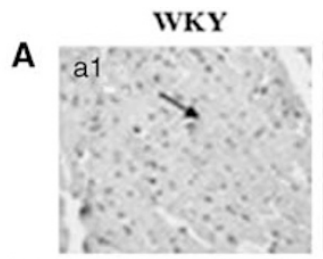

B

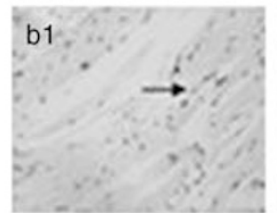

C

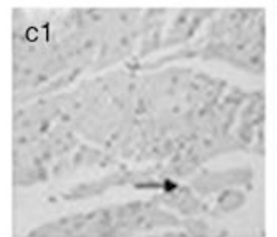

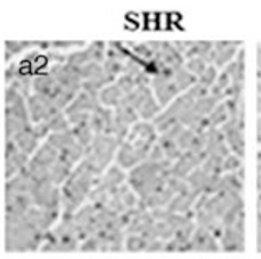
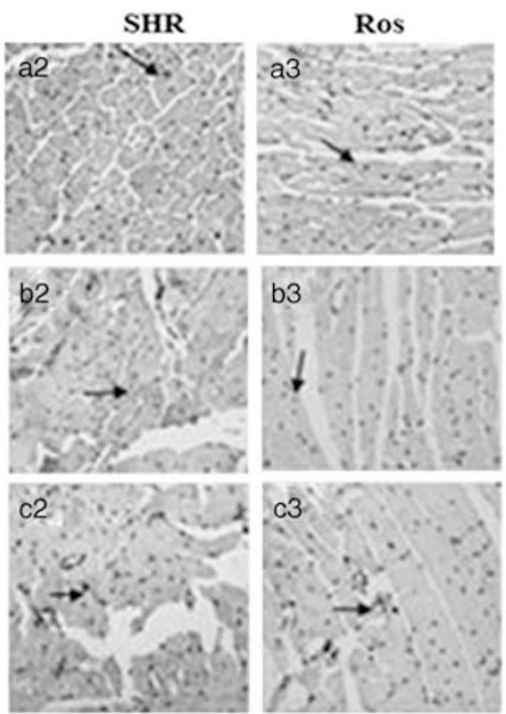

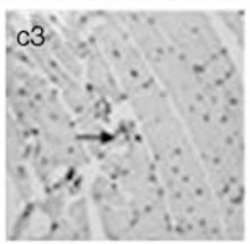

$\mathbf{F}$
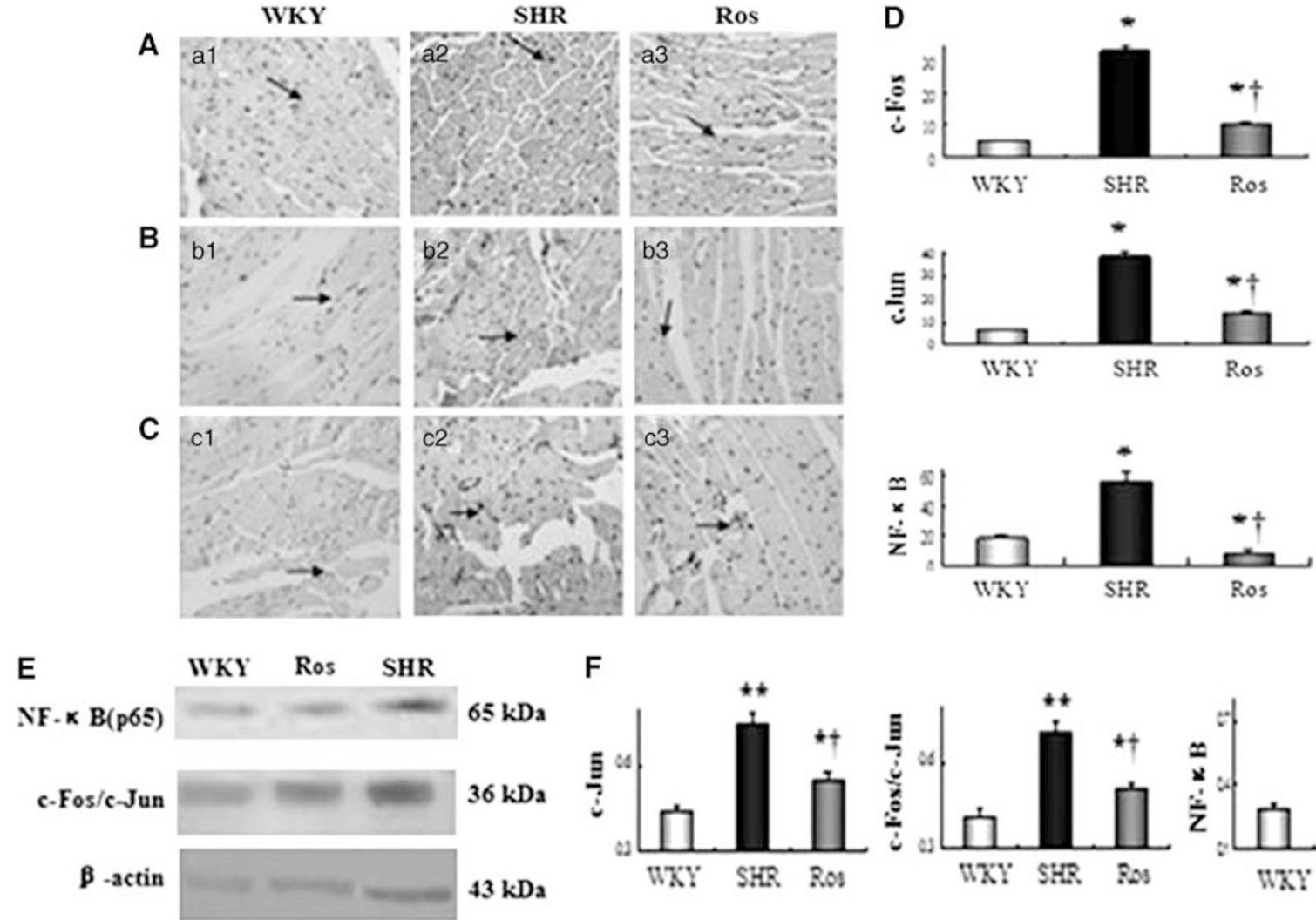

Figure 4 Rosiglitazone administration inhibits protein expression of c-Fos, C-Jun, AP-1 (c-Fos/c-Jun) and nuclear factor-kappa B (NF-kB). Immunostaining for c-Fos, c-Jun and NF-kB in hearts $(\mathbf{A}-\mathbf{C} ; \times 400)$. (a1, b1, c1) Wistar-Kyoto (WKY) rats show few nuclear-positive cells; (a2, b2, c2) spontaneous hypertension rats (SHRs) treated with saline show a large number of nuclear-positive cells; (a3, b3, c3) SHRs treated with rosiglitazone depicting significantly fewer nuclear-positive cells. The scale bar represents $100 \mu \mathrm{m}$. Arrows indicate positive cells. (D) Statistical analysis results of immunostaining for c-Fos, c-Jun and NF-KB. (E, F) Western blot and co-immunoprecipitation analysis of c-Jun, AP-1 (c-Fos/c-Jun) and NF-kB protein expression. Bar graph shows mean \pm s.e.m. of the western blot results. Each assay was performed in triplicate ( $n=5$ per group). ${ }^{*} P<0.05,{ }^{*}{ }^{*} P<0.01$ vs. WKY rats; ${ }^{\dagger} P<0.05$ vs. SHRs.

in the elevated MMP-9/TIMP-1 ratio. Furthermore, the levels of AP-1 and NF- $\mathrm{KB}$ were significantly depressed by rosiglitazone administration. Taken together, this is the first study to demonstrate that rosiglitazone effectively prevented cardiac remodeling by inhibiting AP-1 and NF- $\kappa$ B transcription activity, which may help in further inhibiting the transcription of downstream genes that are involved in the pathogenesis of cardiac remodeling induced by hypertension.

Myocardial remodeling during sustained hypertension is a wellrecognized response of the heart that typically results in both cardiomyocyte hypertrophy and interstitial fibrosis. Cardiac fibrosis is a key phenomenon in hypertensive cardiac remodeling, resulting in increased myocardial stiffness and hence impaired cardiac function. The PPAR- $\gamma$ agonist rosiglitazone is used worldwide for treatment of DM. Does rosiglitazone have a role in essential hypertension? Zhang et al. ${ }^{9}$ demonstrated that SHR cardiomyocytes are insulin resistant, and treatment with rosiglitazone upregulated myocardial PPAR- $\gamma$ expression and restored insulin-induced contractile response in SHRs. Previous studies ${ }^{10}$ have revealed that cardiac-specific PPAR- $\gamma$ deficiency caused modest ventricular hypertrophy but did not impair systolic function. Thus, the effects of PPAR- $\gamma$ agonists on the cardiovascular system have attracted much attention. Yao et al. ${ }^{11}$ were the first to report that PPAR- $\gamma$ agonists could reduce myocardial fibrosis in diabetic rats. Caglayan et al. ${ }^{12}$ reported that PPAR- $\gamma$ agonists attenuate angiotensin II-induced cardiac fibrosis by inhibiting myocardial macrophage infiltration. However, little is known about the role of rosiglitazone in cardiac remodeling evoked by essential hypertension. In our study, we used SHRs as a model to analyze the effects of the PPAR- $\gamma$ agonist rosiglitazone on the development of myocardial remodeling.

To assess the role of rosiglitazone in hypertensive pathological remodeling, we treated SHRs with rosiglitazone for 16 weeks. Rosiglitazone administration significantly reduced the LV mass and LVW/ BW ratio, which were in accord with previous studies. ${ }^{5,13}$ In addition, IVSd and LVPWd markedly decreased compared with SHRs, as assessed by echocardiography. To further verify the effects of rosiglitazone on cardiac fibrosis, we evaluated the standardized IBS and cyclic variation of IBS. The extracellular matrix represents an important source of myocardial IBS, ${ }^{14}$ and several experimental studies have demonstrated that IBS in diastole correlates with the collagen content within the myocardium. ${ }^{15,16}$ Therefore, the acoustic densitometry technique can noninvasively provide a relative estimate of myocardial fibrosis. The standardized IBS and cyclic variation of IBS data were significantly improved in SHRs treated with rosiglitazone when compared with SHRs treated with saline. Furthermore, rosiglitazone treatment significantly reduced collagen I and collagen III mRNA expression. The depression of cardiac remodeling demonstrates the possibility of an early protective effect of rosiglitazone against hypertensive heart damage.

One set of factors involved in the remodeling process is a family of extracellular matrix-degrading enzymes, the MMPs, as well as their endogenous inhibitors, TIMPs. Previous studies ${ }^{17,18}$ have shown that PPAR- $\gamma$ agonists modulate the expression and activity of MMP-9 in human breast cancer cells and macrophages. Our findings indicated 
that hypertension-induced LV hypertrophy was associated with a decreased MMP-9/TIMP-1 ratio. The decreased MMP-9 activity may contribute to ventricular remodeling by impairing the degradation of the interstitial collagens. As expected, rosiglitazone promoted the degradation of the extracellular matrix by increasing the synthesis of MMP-9 and decreasing the activity of TIMP-1. These data suggest that the overall effect of rosiglitazone on MMP-9 and TIMP-1 led to degradation of the extracellular matrix and depression of cardiac fibrosis. That is, rosiglitazone normalized the MMP-9/TIMP-1 ratio, which is a valuable index in the evaluation of fibrosis. ${ }^{19}$ However, the underlying mechanism of rosiglitazone acting on MMP-9/TIMP-1 is not clear.

MMPs and TIMPs are regulated at the transcriptional level by the binding of transcription factors, enhancers and repressors to the gene's promoter region, and their expression is modulated by external stimuli that are operative in cardiac disease states. ${ }^{20}$ MMP-9 and TIMP-1 contain an AP-1 binding site, and MMP-9 has an NF- $\mathrm{BB}$ binding site in its promoter region. In the murine TIMP-1 gene, the AP1 site is located at $-59 \mathrm{bp}$ and is important in both basal and inducible TIMP-1 gene expression. ${ }^{21}$ Many cytokines and matricellular factors influence MMP/TIMP expression through the NF- $\kappa \mathrm{B}$ and AP-1 pathways. $^{22}$ Stimulation of rat cardiac fibroblasts with Ang II induces the production of both NF- $\kappa \mathrm{B}$ and $\mathrm{AP}-1$ transcription factors and is associated with an increase in collagen I production as well as a decrease in MMP-1 expression. ${ }^{23}$ Previous studies ${ }^{24-26}$ have reported that TNF- $\alpha$-induced MMP- 9 expression is regulated by activation of the transcription factors NF- $\mathrm{KB}$ and AP-1. In our study, we found that chronic hypertension induced overexpression of AP-1 and NF- $\kappa$ B. However, the activation of NF- $\kappa$ B and AP-1 was blocked by rosiglitazone administration. The inhibitory effects of rosiglitazone on NF- $\kappa \mathrm{B}$ and AP-1 lead to an elevated MMP-9/ TIMP-1 ratio. Therefore, we considered that rosiglitazone may partially inhibit cardiac fibrosis through the NF- $\kappa \mathrm{B}$ and AP-1 pathways. In addition, there may be many other mechanisms involved in the effects of rosiglitazone on cardiac remodeling. The precise mechanisms through which rosiglitazone modulates MMP-9 and TIMP-1 expression remain unknown.

In conclusion, our results provide evidence for a beneficial effect of rosiglitazone on myocardial remodeling evoked by hypertension in SHRs. Rosiglitazone attenuated cardiac hypertrophy and extracellular matrix accumulation by suppressing NF- $\mathrm{KB}$ and AP-1 activity, resulting in an increased MMP-9/TIMP-1 ratio.

\section{LIMITATIONS}

Further experiments should use the PPAR- $\gamma$ antagonist to establish the cause-effect relationship between rosiglitazone treatment and the beneficial changes on cardiac function and cardiac remodeling, and use PPAR- $\gamma$-overexpressing rats to provide an insight into the precise interactions between PPAR- $\gamma$ and associated signaling pathways involved in the pathogenesis of myocardial remodeling induced by hypertension.

\section{CONFLICT OF INTEREST}

The authors declare no conflict of interest.

\section{ACKNOWLEDGEMENTS}

This work was supported by grants from the Natural Science Foundation of Shandong Province (ZR2009CM026), the Department of Science and Technology of Shandong Province (No. 2006GG2202006), independent innovation fund of Shandong university (2009DX004), the National Natural Science Foundation of China (81070076, 30871038), and the National Basic Research Program of China (973 Program, Grant No.:2010CB732605).
1 Spinale FG. Myocardial matrix remodeling and the matrix metalloproteinases: influence on cardiac form and function. Physiol Rev 2007; 87: 1285-1342.

2 Deng T, Karin M. c-Fos transcriptional activity stimulated by H-Ras-activated protein kinase distinct from JNK and ERK. Nature 1994; 371: 171-175.

3 Li X. Meng Y, Wu P, Zhang Z, Yang X. Angiotensin II and aldosterone stimulating NF-kappaB and AP-1 activation in hepatic fibrosis of rat. Regul Pept 2007; 138: 15-25. 4 Yamamoto K, Ohki R, Lee RT, Ikeda U, Shimada K. Peroxisome proliferator-activated receptor gamma activators inhibit cardiac hypertrophy in cardiomyocytes. Circulation 2001; 104: 1670-1675.

5 Ren L, Li Y, Li Y, Tang R, Hu D, Sheng Z, Liu N. Inhibitory effects of rosiglitazone on cardiac hypertrophy through modulating the renin-angiotensin system in diet-induced hypercholesterolemic rats. Cell Biochem Funct 2010; 28: 58-65.

6 Takahashi R, Asai T, Murakami H, Murakami R, Tsuzuki M, Numaguchi Y, Matsui H, Murohara T, Okumura K. Overload-induced cardiomyopathy in heterozygous carrier mice of carnitine transporter gene mutation. Hypertension 2007; 50: 463-464.

7 Finch-Johnston AE, Gussak HM, Mobley J, Holland MR, Petrovic O, Pérez JE, Miller JG. Cyclic variation of integrated backscatter: dependence of time delay on the echocardiographic view used and the myocardial segment analyzed. J Am Soc Echocardiogr 2000; 13: 9-17.

8 Sun M, Dawood F, Wen WH, Chen M, Dixon I, Kirshenbaum LA, Liu PP. Tumor necrosis factor activation after infarction contributes to susceptibility of myocardial rupture and left ventricular dysfunction. Circulation 2004; 110: 3221-3228.

9 Zhang H, Li J, Li R, Zhang Q, Ma H, Ji Q, Guo W, Wang H, Lopez BL, Christopher TA, Ma X, Gao F. Cardiotropic response to insulin in spontaneously hypertensive rats: role of peroxisome proliferator-activated receptor-gamma-initiated signaling. J Hypertens 2008; 26: 560-569.

10 Duan SZ, Ivashchenko CY, Russell MW, Milstone DS, Mortensen RM. Knockout and agonist of peroxisome proliferator-activated receptor-gamma both induce cardiac hypertrophy in mice. Circ Res 2005; 97: 372-379.

11 Yao L, Mizushige K, Noma T, Murakami K, Ohmori K, Matsuo H. Troglitazone decreases collagen accumulation in prediabetic stage of a type II diabetic rat model. Heart 2000; 84: 209.

12 Caglayan E, Stauber B, Collins AR, Lyon CJ, Yin F, Liu J, Rosenkranz S, Erdmann E, Peterson LE, Ross RS, Tangirala RK, Hsueh WA. Differential roles of cardiomyocyte and macrophage peroxisome proliferator-activated receptor gamma in cardiac fibrosis. Diabetes 2008; 57: 2470-2479.

13 Asakawa M, Takano H, Nagai T, Uozumi H, Hasegawa H, Kubota N, Saito T, Masuda Y, Kadowaki T, Komuro I. Proliferators activated receptor g plays a critical role in inhibition of cardiac hypertrophy in vitro and in vivo. Circulation 2002; 105: 1240-1246.

14 Hall CS, Scott MJ, Lanza GM, Miller LG, Wickline SA. The extracellular matrix is an important source of ultrasound backscatter from myocardium. J Acoust Soc Am 2000; 107: 612-619.

15 Hoyt RM, Skorton DJ, Collins SM, Melton Jr HE. Ultrasonic backscatter and collagen in normal ventricular myocardium. Circulation 1984; 69: 775-782.

16 Picano E, Pelosi G, Marzilli M, Lattanzi F, Benassi A, Landini L, L'Abbate A. In vivo quantitative ultrasonic evaluation of myocardial fibrosis in humans. Circulation 1990; 81: 58-64.

17 Liu H, Zang C, Fenner MH, Possinger K, Elstner E. PPARgamma ligands and ATRA inhibit the invasion of human breast cancer cells in vitro. Breast Cancer Res Treat 2003; 79: 63-74.

18 Marx N, Sukhova G, Murphy C, Libby P, Plutzky J. Macrophages in human atheroma contain PPARgamma: differentiation-dependent PPARgamma expression and reduction of MMP-9 activity through PPARgamma activation in mononuclear phagocytes in vitro. Am J Pathol 1998; 153: 17-23.

19 Bossé M, Chakir J, Rouabhia M, Boulet LP, Audette M, Laviolette M. Serum matrix metalloproteinase-9: tissue inhibitor of metalloproteinase-1 ratio correlates with steroid responsiveness in moderate to severe asthma. Am J Respir Crit Care Med 1999; 159: 596-602.

20 Chakraborti S, Mandal M, Das S, Mandal A, Chakraborti T. Regulation of matrix metalloproteinases: an overview. Mol Cell Biochem 2003; 253: 269-285.

21 Hall MC, Young DA, Waters JG, Rowan AD, Chantry A, Edwards DR, Clark IM. The comparative role of activator protein 1 and Smad factors in the regulation of TIMP-1 and MMP-1 gene expression by transforming growth factor-beta 1 . J Biol Chem 2003; 278: 10304-10313.

22 Deschamps AM, Spinale FG. Pathways of matrix metalloproteinase induction in heart failure: bioactive molecules and transcriptional regulation. Cardiovasc Res 2006; 69: 666-676.

23 Chen K, Chen J, Li D, Zhang X, Mehta JL. Angiotensin II regulation of collagen type I expression in cardiac fibroblasts: modulation by PPAR-gamma ligand pioglitazone. Hypertension 2004; 44: 655-661.

24 Moon SK, Cho GO, Jung SY, Gal SW, Kwon TK, Lee YC, Madamanchi NR, Kim CH. Quercetin exerts multiple inhibitory effects on vascular smooth muscle cells: role of ERK1/2, cell-cycle regulation, and matrix metalloproteinase-9. Biochem Biophys Res Commun 2003. 301: 1069-1078.

25 Moon SK, Cha BY, Kim CH. ERK1/2 mediates TNF-alpha-induced matrix metalloproteinase-9 expression in human vascular smooth muscle cells via the regulation of NF-kappaB and AP-1: involvement of the ras dependent pathway. J Cell Physiol 2004; 198: 417-427.

26 Marx N, Duez H, Fruchart JC, Staels B. Peroxisome proliferator-activated receptors and atherogenesis: regulators of gene expression in vascular cells. Circ Res 2004; 94 : 1168-1178. 